\title{
MVGDRA: Modified Virtual Grid based Dynamic Routes Adjustment Scheme for Mobile Sink-based Wireless Sensors Networks
}

\author{
Navpreet Kaur ${ }^{\mathrm{a}}$, Ashwani Kumar Narula ${ }^{\mathrm{b}}$ \\ ${ }^{a}$ Electronics and Communication Engineering Section, Yadavindra College of Engineering, Punjabi University \\ Guru Kashi Campus, Talwandi Sabo-151302, Punjab, India \\ ${ }^{b}$ Electronics and Communication Engineering Section, Yadavindra College of Engineering, Punjabi University \\ Guru Kashi Campus, Talwandi Sabo-151302, Punjab, India
}

Received: 16 December 2016; Accepted: 25 January 2017; Published: 08 September 2017

\begin{abstract}
In the wireless sensor network, various sensor nodes are present that are used for the communication, sensing and computing. An external mobile sink is present which move around the grid that is used for the communication with the nodes directly which are present in the network. As we know that for formation of network we need to find the route between all the nodes coming in the network, for this purpose routing is done. Routing is defined as moving of information from source to destination. For efficient network the routing protocol that is used should consume less energy, and less distance. In the MVGDRA routing algorithm is proposed that use the mobile sink approach. The selection of cluster head is done on the basis of the weight values. By doing this cluster head will be selected on the basis of the energy means which node has highest energy appointed as cluster head that increases the stability of the network. And the event driven based strategy is used for the data transfer, the energy will be only released when it is required. The energy will not be released after each round. By this the energy consumption of the system decrease and the life time the network is increased. So this method is considered to be more efficient and better than the traditional methods of routing as the network performance is enhanced and its life time is increased. Simulation results show the comparison between the VGDRA and the MVGDRA.
\end{abstract}

Index Terms: Energy efficiency, network life time, mobile sink, wireless sensor networks

(C) 2017 Published by MECS Publisher. Selection and/or peer review under responsibility of the Research Association of Modern Education and Computer Science

* Corresponding author.

E-mail address: manes.nav@gmail.com, ashwaninarula@yahoo.co.in 


\section{Introduction}

A Wi-Fi sensor community (WSN) (once in a while referred to as a wireless sensor and actuator community (WSAN) are spatially disbursed autonomous sensors to screen bodily or environmental situation, which include temperature, sound, strain, and so forth and to cooperatively pass their data via the network to a major vicinity. The more contemporary networks are bi-directional, also permitting manipulate of sensor hobby. The development of Wi-Fi sensor networks turned into stimulated with the aid of military applications consisting of battlefield surveillance, now a day such networks are used in lots of industrial and client programs, consisting of industrial technique tracking and control, machine fitness tracking and so on. Wi-Fi itself organization network for communication devises and a network of a tiny computing device has been generally used in dangerous environment. The WSN is arrangement of "nodes" alien a scattering to join legions or appease thousands, whither ever tumulus is combined to couple (or rarely duo) sensors. Many nodes are placed in the grid network which are communicates the other node lie outside the grid in the network. The basically outside node is called sink where nodes shows to $n$-to- 1 communication in reporting a observe date by a single sink [1].The efficiency of network depends upon the selection of the path as it also effects the life time of network and the energy consumption. Various routing algorithms have been designed for the efficient routing process. Link state routing and Distance vector routing are two most popular dynamic routing algorithm used in wireless networks. The main drawback of these routing is the slow convergence. The wireless sensor networks are accurate, cost effective, reliable and easily deployed. The life time of network is determined by the energy consumed by the sensor node [2].

Routing is defined as moving of information from source to destination. For efficient network the routing protocol that is used should consume less energy, and less distance. As in virtual gridding protocol, the sink was kept mobile and the cluster head selection was done on the basis of the distance of nodes from the center. But this method was cluster head selection was not efficient so after studying the literature a new method of the cluster head selection is proposed in this work. The cluster head selection is done on the basis of the weight values. The weight values will help in the selection of the cluster head from the cluster of the node. By doing this cluster head will be selected on the basis of the energy that the result in increasing the stability of the network. Secondly in traditional approaches each node transmits energy after every round that results in the more energy consumption and thereby decreasing the life time of the network. So to resolve this problem the event driven based strategy is used for the data transfer, the energy will be only released when it is required. The energy will not be released after each round. By this the energy consumption of the system decrease and the life time the network is increased. So this method is considered to be more efficient and better than the traditional methods of routing as the network performance is enhanced and its life time is increased. Simulation results reveal decreased energy consumption and increased life time of network to compare the VGDRA.

The rest of this paper is organized as follows: Section 2 described the related work include the various approaches that deal the data delivery to a mobile sink in WSN. Section 3 presents our MVGDRA scheme in details. The simulation results and setup are present in Section 4. Finally, Section 5 represents the conclusion the paper.

\section{Related Work and Protocols}

In this it creates a virtual infrastructure by partitioning the sensor field into a virtual grid of a uniform cell size. The aim of this scheme to minimized the energy consumption and increased the life time of network. This scheme supports only straight line communication. Unlike the static sink sensor, the network topology become a dynamic as the sink keeps changing own position. The several infrastructure based in the data dissemination protocol have been suggest for mobile sink based wireless sensor network. In the sensor field, mobile pattern based or shows by the sink, the data dissemination or collection scheme can be classified into two patterns, which are un-controlled and controlled sink mobility scheme. The uncontrolled mobility scheme based on the 
facts by that the sink makes its next move independently in term of speed and direction. The controlled mobility scheme, the direction or speed of sink is controlled and the manipulated by the external observer [3]. Data dissemination is the estimated method of data collect or distribute among the nodes of the network. Several virtual infrastructures are based on the data dissemination protocols in which the mobile sink based on the wireless network. This paper consider the several of protocols and the uncontrolled sink mobility environments have been discussed below

\subsection{Virtual circle combined straight routing (VCCSR)}

In VCCSR, a virtual structure is designed having straight lines and virtual circles. Along these straight lines and virtual circles, a set of nodes are appointed as a $\mathrm{CH}$ (cluster head). In the sensor field the sink will circulate and it will collect the information. In the VCCSR, cluster heads $(\mathrm{CH})$ follow a set of communication rules are defined to minimizing the routes reconstruction cost that based on the latest location of sink. In this protocol the reconstruction cost is minimize but due to the cluster head present in center of sensor field can collect the different amount of energy so it can cause decrease in its energy communication in the network [1].

\subsection{Hexagonal cell-based data dissemination (HexDD)}

In this, the hexagonal grid structure constructs a multiple numbers of mobile sink for the data transmission. For defining the data location and query the data is based on the six different direction of a hexagon. It can also avoid the redundant propagation of sink data. Nodes send the data to nearest border line then it distribute the data to center line. At the border line nodes are store and replicate the data. When the sink is moved from one cell to another it inform the current location to border line nodes and the center nodes. In this way if the speed of the mobile sink is more than it consumes more energy [1].

\subsection{Backbone-based virtual infrastructure (BVI)}

It is the data dissemination scheme in which single level multi hop clustering is used. The aim of this infrastructure to decreases the number of cluster. So that every cluster heads should know the location of sink in which it will set its route. But it has one drawback that the life time of network is decreases [2], [11].

\subsection{Multiple enhanced specified-deployed sub-sinks (MESS)}

It generates a virtual strip in the middle of sensor field. It has more storage capacity at equal distance which improved the position of (sub-sinks) wireless nodes. The set of sub-sink nodes along the approachable path in which it serve as meeting point for the mobile sinks. Mobile sink collect and store the data from sensor nodes. Mobile sink move the query along the virtual strip till it reaches to the sub sink node owning the data during the data delivery phase. Using the geographical forwarding approach the query is received from mobile sink and the sub sinks route their deposited data to the mobile sink [3].

\section{The MVGDRA Scheme}

In Modified Virtual Grid Dynamic Route Adjustment Scheme, it includes how to construct the infrastructure and maintain the routes toward the latest location of the mobile sink. The sensor area partitioned into a equal cells size of grid. Cell head is present in each cell to manage the all other sensors with in cell. The total number of cells is a function of the number of sensor nodes. A node which has a highest energy to the other nodes is appointed as a cell header in each cells. Which are responsible for keeping track of the latest location of the mobile sink. By doing this cluster head will be selected on the basis of the energy that resulted in increasing the 
stability of network. The adjacent cell-headers exchange information via gateway nodes. The set of cell-header nodes together with the gateway nodes constructs the virtual backbone structure. In MVGDRA, each node will not transmit energy after every round. The energy will be only released when it is required. By this the energy consumption of the system decrease and the life time is increased.

\subsection{Network characteristics and formation}

The following network characteristics are assumed for operation of MVGDR scheme.

- Nodes are randomly deployment remain static.

- All the nodes are homogeneous architecture and acquire location information.

- Transmission power of nodes based on the distance to the destination nodes.

- All the sensor nodes, the throughout remain static and have same energy level whereas the mobile sink does not have any resource constraints.

- A single mobile sink performs periodic data collection.

This scheme constructs a virtual grid by partitioning the sensor field into the several uniform sized cells based on the number of nodes in the sensor field. The aim behind this scheme, to increases the life time of network and decreases the energy consumption of network.

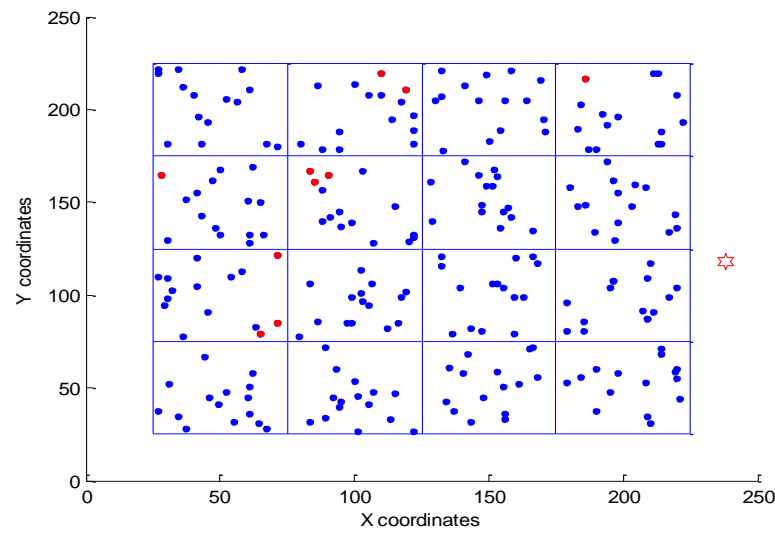

Fig.1. Virtual grid structure of modified virtual grid based dynamic route adjustment in wireless sensor network

The Fig.1.shows the virtual gridding based dynamic routing. In this the red color star represents a mobile sink is move around the grid in the given network, which communicate with the cluster head of each cell. The cluster head selection is done on the basis of the weight values means which has high energy is appointed as cluster head. The blue color dots are in the given network represents a number of nodes and red color dots are represents a number of dead nodes.

\subsection{Cell-head rotation}

An important part of the MVGDRA scheme is rotating the role of cell head to every node in the cell. Cell heads are normal nodes with the same characteristics of other sensor nodes. Cell head collect the data from other nodes and forward to the mobile sink. The cluster head selection is done on the basis of the weight values. 
The weight values will help in the selection of the cluster head from the cluster of the node. By doing this cluster head will be selected on the basis of the energy that means which node has high energy is appointed as cluster head that the result in increasing the stability of the network.

\section{Simulation and Results}

In this section, we present the simulation result using MATLAB. The total area of the grid sensor filed network is 200 and total number of nodes in each cluster is 15. Mobile sink moves around the grid field counterclockwise. We creates another network around the grid which shown in figure1. The distance between virtual networks from the grid is 25 therefore mobile sink lie in between this network. The initial point of the mobile sink is 13 and end point is 238 . Therefore $\mathrm{X}=13$ and $\mathrm{Y}=238$.

Furthermore, we consider nodes energy consumption in transmission (Tx) and receiving (Rx) nodes which are calculated by using following equation (1).

$$
\begin{aligned}
& T x=\left(E_{\text {elect }} * k\right)+\left(E_{\text {amp }} * k * d^{2}\right) \\
& R x=E_{\text {elect }} * k
\end{aligned}
$$

In our experiment, we used $E_{\text {elect }}=50 \mathrm{nj}$, and $\mathrm{E}_{\mathrm{amp}}=10 \mathrm{~nJ} / \mathrm{bit} / \mathrm{m}^{2}$ and $\mathrm{K}=8 \mathrm{bits}$. Where $\mathrm{E}_{\text {elect }}$ is the nodes energy dissipation in order to run its radio electronic circuitry and $\mathrm{E}_{\mathrm{amp}}$ is the energy dissipation by the transmitter amplifier and $\mathrm{K}$ is the message length, $\mathrm{d}$ is the distance between sender and receiver. The total number of packet is equal to the $8 * 2000$.

In this section we also compare our MVGDRA scheme with VGDRA where a common feature among them is the use of a virtual infrastructure for network operation.

\subsection{The energy of network communication}

The energy of network communication represents the energy of nodes which are used for the communication between the nodes with mobile sink. In this we compare the energy of network VGDRA and MVGDRA. In the VGDRA, the cluster head selection was done on the basis of the distance of nodes from the center. But this method was cluster head selection was not efficient so after studying the literature a new method of the cluster head selection is proposed in the MVGDRA. By doing this cluster head will be selected on the basis of the energy means which node has highest energy with the others is appointed as cluster head that result in increasing the stability of the network. In the Fig.2.we show the energy of network is different in VGDRA and MVGDRA.

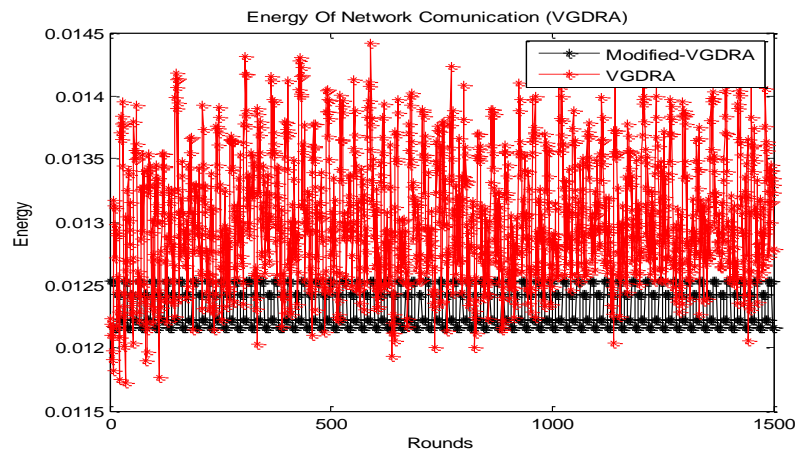

Fig.2. Comparing the energy of network communication between the VGDRA and MVGDRA. 


\subsection{The first dead node}

It is defined as the time since the nodes deployment till the first node dies due to energy depletion. In the VGDRA the each node transmits energy after every round that results in the more energy consumption and thereby decreasing the life time of the network. So to resolve this problem, in MVGDRA the event driven based strategy is used for the data transfer, the energy will be only released when it is required. The energy will not be released after each round. By this the energy consumption of the system decrease and the life time the network is increased.

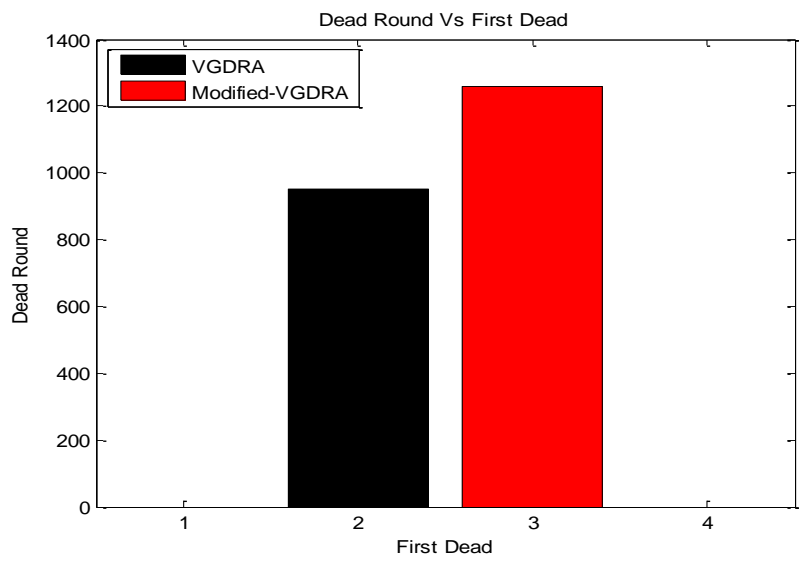

Fig.3. Dead round Vs first dead node in VGDRA and MVGDRA

The Fig.3.shows, in after how many round the first dead node occur in VGDRA and MVGDRA. We discussed in above, due to the more energy consumption in VGDRA the dead node occur quickly so that it occur after the 900 rounds approx. Same in MVGDRA, dead node occur after 1200 rounds because it has less energy consumption so that life time the network increased.

\subsection{The alive nodes and dead nodes Vs number of rounds}

In this we discuss about the nodes are alive in how much round and after that, how much round the nodes are dead. The following figure shows alive period and dead period of nodes. We also compare the alive nodes and dead nodes of VGDRA with MVGDRA. In MVGDRA, approximately 10 number of dead node occur but in VGDRA, the number of dead nodes more than the MVGDRA, which are shown in Fig.4. and similarly alive nodes present at how many round which shown in Fig.5. Because Modified virtual grid based dynamic route adjustment has less energy consumption so that life time the network increased. In this the red color represents the nodes are alive during the 1200 rounds and nodes are dead after the 1200 rounds in the modified virtual grid based dynamic route adjustment and black color represents the nodes are dead and alive after the 900 rounds in virtual grid based dynamic route adjustment scheme for mobile sink based wireless sensor networks. Fig.4. show the dead nodes only along the number of rounds in virtual infrastructure network. The $\mathrm{X}$ coordinate represents the number of rounds and $\mathrm{Y}$ coordinate represents the number of dead nodes in both networks means in modified virtual grid based dynamic route adjustment and virtual grid based dynamic route adjustment. The dead nodes in virtual grid based dynamic route adjustment occur faster than the modified virtual grid based dynamic route adjustment. 


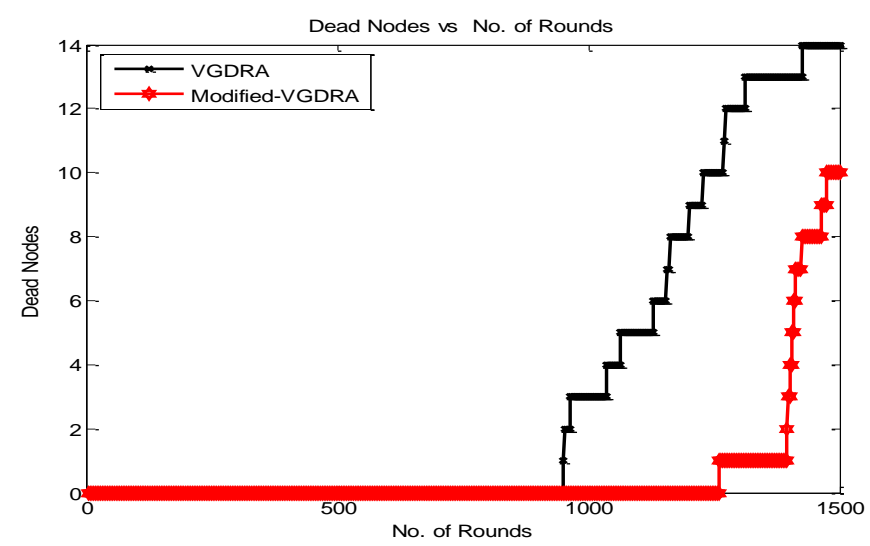

Fig.4. The dead nodes Vs number of rounds in VGDRA and MVGDRA.

Fig.5. shows the alive nodes only along the number of rounds in virtual infrastructure network. The $X$ coordinate represents the number of rounds and $\mathrm{Y}$ coordinate represents the number of alive nodes in both networks means in modified virtual grid based dynamic route adjustment and virtual grid based dynamic route adjustment. The alive nodes in virtual grid based dynamic route adjustment occur lesser time than the modified virtual grid based dynamic route adjustment. In the virtual grid based dynamic route adjustment, the alive nodes occur along in the 900 rounds but in case of modified virtual grid based dynamic route adjustment, the alive nodes occur along the 1200 rounds in the given network. In MVGDRA, approximately 10 number of dead node occur but in VGDRA, the number of dead nodes more than the MVGDRA. Because Modified virtual grid based dynamic route adjustment has less energy consumption so that life time the network increased.

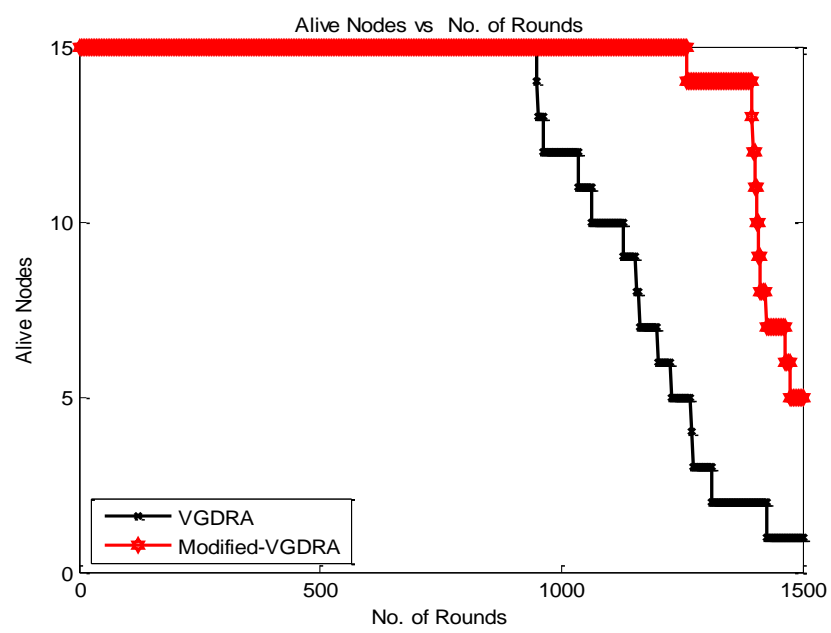

Fig.5. The alive nodes Vs number of rounds in VGDRA and MVGDRA. 


\section{Conclusions and Future Work}

The sensor field into a virtual grid and constructs a communication path through the cell header to mobile sink. A mobile sink while moving around the sensor field keeps on changing its location and interacts with the highest energy cluster head for data collection. That result in increasing the stability of the network. Using a set of communication rules only limited number of cell header take part in the routes reconstruction process. The event driven strategy used for the data transfer, the energy will be released when it is required. By this the energy consumption of the system decrease and the life time the network is increased. In this, it also shows the dead nodes in virtual grid based dynamic route adjustment occur faster than the modified virtual grid based dynamic route adjustment. In the virtual grid based dynamic route adjustment, the dead nodes occur after 900 rounds but in case of modified virtual grid based dynamic route adjustment, the dead nodes occur after 1200 rounds in the given network and in the (MVGDRA) Modified virtual grid based dynamic route adjustment, approximately 10 number of dead node occur but in the virtual grid based dynamic route adjustment (VGDRA) the number of dead nodes more than the MVGDRA. So this method is considered to be more efficient and better than the traditional methods of routing as the network performance is enhanced and its life time is increased. In term of nodes energy consumption, the simulation result reveal improved the performance of network.

In the future work, we will use different parameters for the selection of cluster head and analyze the performance of VGDRA scheme at different sink's speeds and different data generation rates of the sensor nodes. The different parameters like selection of cluster head on the basis of distance from the mobile sink and node to node, on the basis of speed and life time of the node. By doing this may also improve the life time of network and reduce the reconstruction cost even more. Energy consumption may also be reduced by using the distance priority communication.

\section{References}

[1] Abdul Waheed Khan, Abdul Hanan Abdullah, "A Virtual Grid-Based Dynamic Routes Adjustment Scheme For Mobile Sink”, IEEE sensors journal, vol.15.Jan. 2015, pp. 526-534.

[2] Jitin Rajan, Abhishek Gupta, "Virtula Grid Based Dynamic Routing Protocol”, Computer science \& tech., vol.4.jun.2016, pp. 158-160.

[3] Sincy Thomas, Syamamol T, "Efficient Data Access in WSN Using AVGDRA Scheme", Compt. Networks, vol.6. jun.2016, pp.148-151.

[4] Shipla Jaswal, Abhitesh, "A Review On Virtual Grid Based Dynamic Routes Adjustment In WSN", International Research Journal of Engineering and Technology, vol.3.feb.2016, pp.957-960.

[5] Gadekar Vaishali, “A Virtual Grid-Based Dynamic Routes Adjustment Scheme For WSN Based on Sink Mobility", International Journal of Innovative Research in Compt. And Comm. Engineering, vol.3.Nov.2015, pp.12033-12037.

[6] Abdul Waheed Khan, "VGDD: A Virtual Grid Based Data Dissemination Scheme for Wireless Sensor Networks with Mobile Sink", International Journal of Distributed Sensor Networks, vol.2015, pp. 1-17.

[7] Amith Khandakar (2012) "Step by Step Procedural Comparison of DSR, AODV and DSDV Routing protocol”, IPCSIT, vol.40, pp 36-40.

[8] Abdul Waheed Khan (August, 2015) "VGDD: A Virtual Grid Based Data Dissemination Scheme for Wireless Sensor Networks with Mobile Sink", International Journal of Distributed Sensor Networks Volume 2015, pp 1-17.

[9] Er. Shilpa jaswal (Feb, 2016) "A Review on Virtual Grid based Dynamic Routes Adjustment in WSN", Vol:03, Issue:02, pp 957-960. 
[10] Jieun Cho (Jan, 2009)“A Routing Protocol using Relative Landmark based on Virtual Grid in Wireless Sensor Network", IEEE, pp 1-3.

[11] Jin Wang (2014) "A Survey about Routing Protocols with Mobile Sink for Wireless Sensor Network", International Journal of Future Generation Communication and Networking Vol:7, No.5 , pp.221-228.

[12] Priya Maidamwar (october, 2012) "A Survey on Security Issues To Detect wormhole attack in Wireless Sensor Network, IJANS, Vol:2, No.4.

\section{Authors' Profiles}

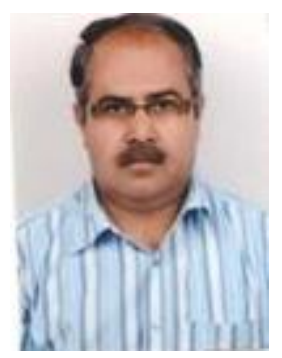

Ashwani Kumar Narula was born in 1970 at Faridkot, Punjab, India. He received the Bachelor of Engineering degree in Electronics from Marathwada University, Aurangabad, Maharashtra, India in 1992 and Master of Engineering in Electronics and Communication Engineering from Thapar Institute of Engineering and Technology a deemed University (now Thapar University), Patiala, Punjab, India in 2001. He also got the Ph.D. degree from Sant Longowal Institute of Engineering and Technology, Longowal, Sangrur, Punjab, India in 2016. He is working as an Associate Professor in Electronics and Communication Engineering Section at Yadavindra College of Engineering, Punjabi University, Guru Kashi Campus, Talwandi Sabo, Punjab, India. His areas of interests are in artificial neural Networks, fuzzy logic, virtual instrument and wireless sensors. He is life member of Indian Society of Technical Education (ISTE) and International Association of Engineers (IAENG).

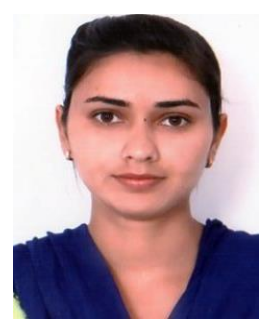

Navpreet Kaur was born in 1992 at Zira, Punjab, India. She received the bachelor of technology degree in Electronics and Communication Engineering from Adesh institute of Engineering \& Technology, Faridkot, Punjab, India in 2014. She is pursuing Masters of technology in Electronics and Communication Engineering from Yadavindra College of Engineering, Talwandi Sabo, Punjab, India.

How to cite this paper: Navpreet Kaur, Ashwani Kumar Narula," MVGDRA: Modified Virtual Grid based Dynamic Routes Adjustment Scheme for Mobile Sink-based Wireless Sensors Networks", International Journal of Wireless and Microwave Technologies(IJWMT), Vol.7, No.5, pp. 40-48, 2017.DOI: 10.5815/ijwmt.2017.05.05 